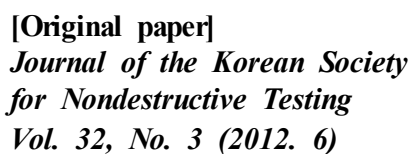

\title{
Thermographic Detection of Surface Crack Using Holomorphic Function of Thermal Field
}

\author{
Nohyu Kim*† and Zongsoo Lim**
}

\begin{abstract}
This paper describes an analytic method for infrared thermography to detect surface cracks in thin plates. Traditional thermographic method uses the spatial contrast of a thermal field, which is often corrupted by noise in the experiment induced mainly by emissivity variations of target surfaces. This study developed a robust analytic approach to crack detection for thermography using the holomorphic function of a temperature field in thin plate under steady-state thermal conditions. The holomorphic function of a simple temperature field was derived for 2-D heat flow in the plate from Cauchy-Riemann conditions, and applied to define a contour integral that varies depending on the existence and strength of singularity in the domain of integration. It was found that the contour integral at each point of thermal image reduced the noise and temperature variation due to heat conduction, so that it provided a clearer image of the singularity such as cracks.
\end{abstract}

Keywords: Thermography, Holomorphic Function, Surface Crack, Thermal Noise, Heat Flow

\section{Introduction}

Active thermography including optically or inductively heated thermography offers a non-contact and rapid inspection method that detects flaws set in a plane parallel to the surface such as delaminations in multilayered structures like composite materials. Active thermography technique has been employed successfully to increase its sensitivity and image contrast of thermography for a specimen that has nonhomogeneous emissivity or irregular surface. However, the conventional approach of active heating a wide area of an object by high power flash lamp is not effective for the detection of surface crack that develops perpendicular to the surface. The detection of such cracks in metallic components is a major concern for the steel manufacturing and automobile industry. Even though other traditional NDT techniques such as ultrasound and eddy current are used in the industry, there has been an increasing interest in investigating the potential of thermography-based techniques because they provide more rapid, efficient and convenient inspection methods. Infrared thermography techniques (IRT) combining thermography with laser scanning or pulsed eddy current have been studied to detect surface cracks. Sakagami [1,2] introduced a new induction IRT technique termed "singular method" for crack identification. In the singular method, a crack is identified from the singular electro-thermal field generated around the crack tips, where the eddy-current density concentrates and leads to temperature rise. While applying a periodically modulated electric current to the cracked sample, the intensity of the singular current field was

[Received: May 11, 2012, Revised: June 7, 2012, Accepted: June 8, 2012] *Department of Mechatronic Engineering, Korea University of Technology and Education, 307 Gajun-Ri, Byungchun-Myun, Chunan 330-860, Korea, **NDE Division, Research Institute of Industrial Science and Technology, Pohang 790-330, Korea †Corresponding Author: nykim@kut.ac.kr 
recorded in the same frequency. This cyclic change of singular electro-thermal field causes temperature distribution cyclically, which can be imaged by the lock-in mode using the reference signal of modulated electric current. Almond [3], Nezelmann [4], and Zenzinger [5] calculated the current distribution induced by an external coil to investigate current flow near the surface of specimen. The current density produced at the vicinity of the crack was visualized by measuring the temperature field. They detected the crack from direct observation of the heating process due to the concentrated current density near crack. Holland [6] visualized the surface crack by employing vibration-induced frictional heating of a crack in titanium. A laser scanning technique combined with thermography was also developed and proved to be very successful in finding the surface crack by $\mathrm{Li}$ [7]. The laser scanning method used the laser as the heat source, for example, $\mathrm{Nd}: \mathrm{YAG}$ laser for local excitation of specimen, and recorded the resulting temperature field with an infrared camera. Based on this data, crack-caused anisotropies in the lateral heat flow was detected and exploited to characterize the crack. Another approach to detect a surface crack is the forced diffusion thermography proposed by Lesniak [8]. It projected a pattern of dynamic heat to generate a heat flow across the crack thereby optimizing the measureable thermal gradient. Basic idea of detecting a crack is that the heat flow is impeded by the crack creating an abrupt change in the temperature, so that the thermal gradient can define the crack. However, the derivative of thermal image may magnify the noise from many sources so much that the gradient image does not always provide better quality in contrast than raw thermal image.

This paper proposes a new analytic method to define and locate surface cracks from noisy thermal images obtained in thin plates. This method uses the holomorphic function of a temperature field in the plate under steady-state thermal condition to enhance crack profile out of noisy image. Theoretical holomorphic function was derived for 2-D heat flow in the plate from Cauchy-Riemann conditions, and applied to define contour integral. The contour integral has an interesting feature that it varies very much depending on the existence and strength of singularity in the domain of integration. An artificial crack-like singularity was created in the plate model to investigate the performance of the proposed method.

\section{Heat Equation in Plate}

Heat conduction in a thin plate is governed by the two dimensional heat conduction (diffusion) equation in absence of heat source in the domain [9],

$$
\begin{aligned}
& \frac{\partial T(x, y, t)}{\partial t}=\alpha \cdot \nabla^{2} T(x, y, t)=\alpha\left[\frac{\partial^{2} T(x, y, t)}{\partial x^{2}}+\frac{\partial^{2} T(x, y, t)}{\partial y^{2}}\right] \\
& \alpha=\frac{k}{\rho c_{p}} \text { (thermal diffusivity) }
\end{aligned}
$$

where $\mathrm{T}$ is temperature, $\mathrm{k}$ is thermal conductivity, and $\alpha$ is thermal diffusivity. In the time-independent case, i.e., $\frac{\partial T}{\partial t}=0$, the heat equation of eqn. (1) reduces to

$$
\nabla^{2} T(x, y, t)=\left[\frac{\partial^{2} T(x, y, t)}{\partial x^{2}}+\frac{\partial^{2} T(x, y, t)}{\partial y^{2}}\right]=0
$$

This Laplace equation of eqn. (2) is satisfied wherever in the domain the steady state is reached without heat source. Exact solution to eqn. (2) is well known as the holomorphic function (or analytic function) in complex analysis. If the temperature distribution $T(x, y)$ is the real valued function of a complex function $f(x+i y) \quad$ such as $f(x+i y)=T(x, y)+i \Theta(x, y)$, $T(x, y)$ automatically satisfies the Laplace 


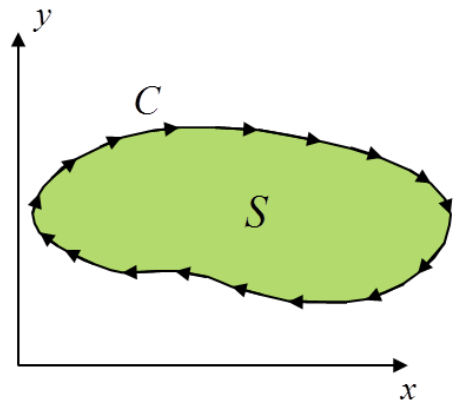

Fig. 1 Contour integral along $\mathrm{C}$

equation in eqn. (2) only if the function $f(x+i y)$ is analytic. In this complex function $f(x+i y), \Theta(x, y)$ is the conjugate harmonic function of $T(x, y)$. The Cauchy-Riemann equations for the function $f(x+i y)$ is given by

$$
\frac{\partial T}{\partial x}=\frac{\partial \Theta}{\partial y}, \frac{\partial T}{\partial y}=-\frac{\partial \Theta}{\partial x}
$$

From the above equation (3), the conjugate harmonic function $\Theta(x, y)$ is determined in terms of $T(x, y)$ by

$$
d \Theta(x, y)=-T_{y}(x, y) d x+T_{x}(x, y) d y
$$

Let $f(z)=f(x+i y)$ be a holomorphic function defined over a domain $\mathrm{S}$ inside the plate. Then the following equation always holds for any contour in the domain $\mathrm{S}$ in Fig. 1,

$$
\int_{C} f^{\prime}(z) d z=d f(z)=0
$$

This integrability condition of eqn. (5) implies that the contour integral surrounding the domain $\mathrm{S}$ as shown in Fig. 1 becomes zero. This condition is always valid locally unless the line $\mathrm{C}$ does not loop around any singularity.

Moreover, by Stokes' theorem, eqn. (5) is simplified to

$$
\int_{C} d \Theta=0
$$

Eqn. (6) is true only if the domain $\mathrm{S}$ does not contain any singularity. But if it has any singularity inside, the contour integral have non-zero value. The singularity may be any discontinuity like crack or heat source. This property of a holomorphic function is the basic concept to find and enhance the singularity in this work.

\section{Numerical Simulation for the Detection of Artificial Crack}

In order to investigate the characteristics of eqn. (6) in a two-dimensional plate of steel, we started with a simple solution to the Laplace equation in 2-D, which is,

$$
T(x, y)=\sin y \cdot \sinh x
$$

This temperature field depicted in Fig. 2(a) satisfies eqn. (6) naturally. Then an artificial random noise signal was added to the temperature field $T(x, y)$ in eqn. (7) to simulate emissivity variance of surface in experiment, which is represented in Fig. 2(b). The maximum value of random noise was set to the median of $T(x, y)$.

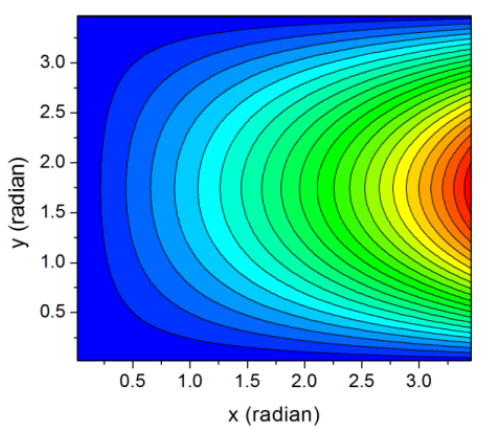

(a)

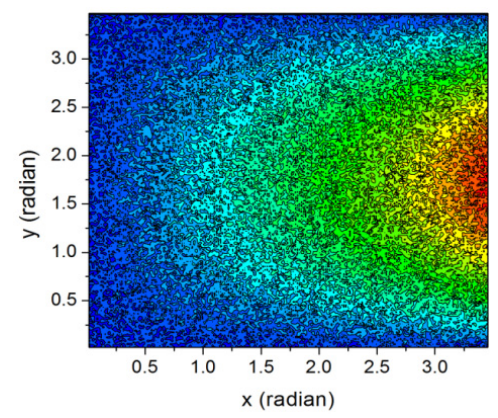

(b)

Fig. 2 Temperature distribution of (a) $T(x, y)=$, $\sin y \cdot \sinh x$ (b) noise-corrupted $T(x, y)$ 
At each point of pixel in the uncorrupted image of Fig. 2(a), contour integral was calculated from eqn. (6) along a closed loop surrounding the point of pixel, and represented in the trimetric diagram of Fig. 3(a). In Fig. $3(a)$, the integral values are very small (approximately zero) at all points $(\mathrm{x}, \mathrm{y})$ of the plate because it has no singularity. What the contour integral means in Fig. 3(a) and (b) is a temperature discontinuity or jump caused by singularity in the thermal field. Thus the higher the integral value is, the stronger the singularity

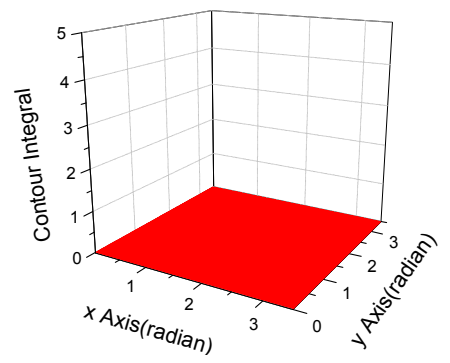

(a)

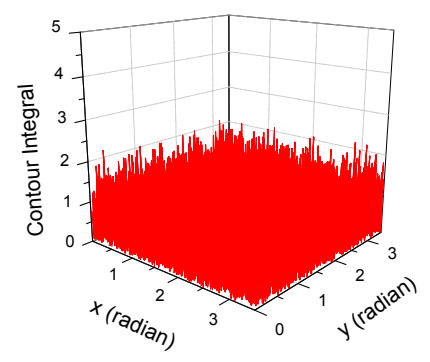

(b)

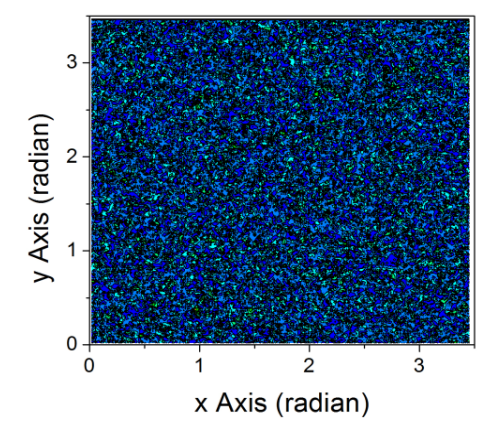

(c)

Fig. 3 Contour integral image of (a) temperature field $T(x, y)=\sin y \cdot \sinh x, \quad$ (b) noisecorrupted $T(x, y)$, (c) the plan view of (b) becomes. In the same manner, the contour integration of the corrupted temperature field in Fig. 2(b) was conducted using eqn. (6) and shown in Fig. 3(b). The plan view of Fig. 3(b) is plotted again in Fig. 3(c) to show the distribution of integral value over the region, where any particular pattern or tendency cannot be observed. These results indicate that the contour integration of a temperature field generated by a steady-state heat flow in a thin plate gets rid of the effects of heat flow on the temperature distribution. This characteristic of contour integral is important to increase signal-to-noise ratio(SNR) of noise-corrupted thermal image.

In addition to the noise, a slit of singularity was imbedded in the temperature field $T(x, y)$ of Fig. 2 to simulate the effect of crack on the

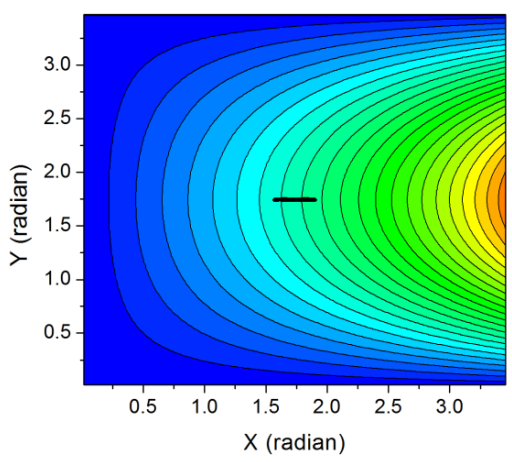

(a)

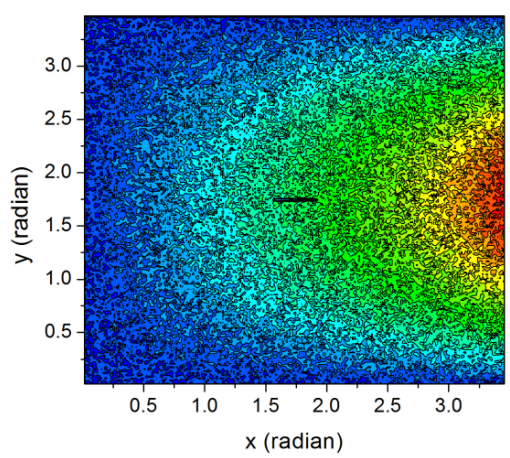

(b)

Fig. 4 A singular crack imbedded in (a) a field $T(x, y)=\sin y \cdot \sinh x$, (b) noise-corrupted $T(x, y)$ 


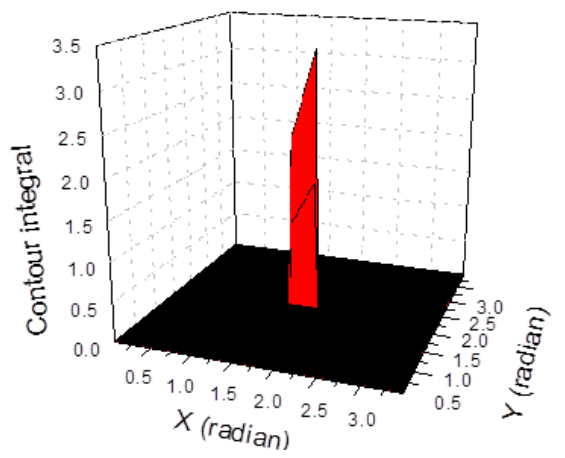

(a)

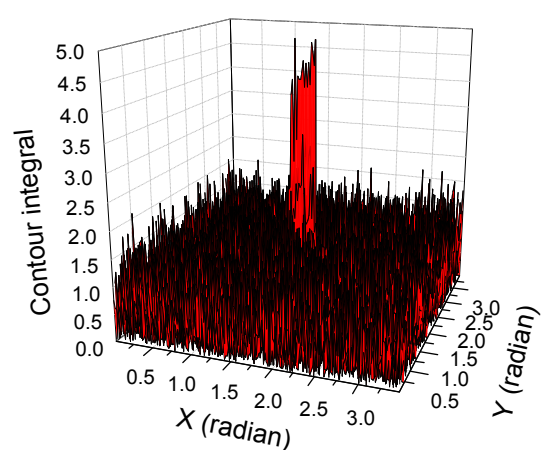

(b)

Fig. 5 Contour integral image of (a) temperature field $T(x, y)$, (b) noise-corrupted $T(x, y)$

integral of eqn. (6). Fig. 4(a) is the temperature distribution of the plate with no noise, and Fig. $5(\mathrm{~b})$ is of a plate with the same noise as in Fig. 4(b). The integral calculation over the temperature images shown in Fig. 4(a) and 4(b) was performed and represented respectively in Fig. 5, where the singular area (crack) is clearly seen in both cases. From these figures, it is found that the analytic method using the contour integral given in eqn. (6) has ability to select and detect the singularity very well because it suppresses both of the temperature variances due to heat conduction and noise.

\section{Conclusions}

A new analytic method in infrared thermography using the contour integral of holomorphic function was proposed to detect surface cracks in a thin plate based on Cauchy-Riemann conditions of steady-state thermal field. A holomorphic function, which is the conjugate harmonic function of temperature field, was derived and proved in theory that its contour integral became zero over a domain where there was no singularity. On the contrary, if the domain had any singular point like a crack, the contour integral produced non-zero value. Based on this property of contour integral, a simple 2-D model for steady-state heat conduction was created and tested to estimate by numerical analysis the performance of the proposed contour integral method for crack detection. A severe random noise and slit-like crack were also added to the temperature field of the model to investigate how well the integral image detects singularity. The integral image showed that the contour integral reduced not only the variations of temperature produced by heat flow but also noise, so that it selected and detected crack excellently and improved image contrast.

\section{Acknowledgments}

Authors gratefully acknowledge the support to this work partly by Korea University of Technology and Education, Ministry of Educational Science and Technology(MEST), and Research Institute of Advanced Science and Technology(RIST).

\section{References}

[1] T. Sakagami and S. Kubo, "Development of a new non-destructive testing technique for quantitative evaluations of delamination defects in concrete structures based on phase delay measurement using lock-in thermography," Infrared Physics \& Technology, Vol. 43, pp. 211-218 (2002)

[2] T. Sakagami and S. Kubo, "Development 
of a new crack identification technique based on near-tip singular electrothermal field measured by lock-in infrared thermography," JSME International Journal, Series A, Vol. 44, No. 4, pp. 528-534 (2001)

[3] I. Z. Abidin, G. Tian, J. Wilson, S. Yang and D. Almond, "Quantitative evaluation of angular defects by pulsed eddy current thermography," NDT \& E International 43(7), pp. 537-546 (2010)

[4] G. Walle and U. Netzelmann, "Thermographic crack detection in ferritic steel components using inductive heating," Proc. of ECNDT, Tu.4.8.5 (2006)

[5] G. Zenzinger et al., "Thermographic crack detection by eddy current excitation," Nondestructive Testing and Evaluation, 22, pp. 101-111 (2007)

[6] S. D. Holland and J. Renshaw, "Physicsbased image enhancement for infrared thermography," NDT\&E International 43(5), pp. 440-445 (2010)

[7] T. Li, D. P. Almond and D. A. S. Rees, "Crack imaging by scanning pulsed laser spot thermography," NDT\&E International 18(1), pp. 117-123 (2011)

[8] J. R. Lesniak and D. J. Bazile, "Forceddiffusion thermography technique and projector design," Proc. SPIE Vol. 2766, pp. 210-217 (1996)

[9] T. Lee, N. Kim and J. Lim, "Thermographic detection of surface crack," Fall conference Proc. of Korean Society for Nondestructive Testing, pp. 241-246 (2011) 